Journal of Case Reports 2021;11(1):12-15

\title{
Splenic Rupture in an Obstructed Colorectal Cancer
}

\author{
O Shams, F Ftaieh, MA Gok, MM Sadat, UA Khan \\ East Cheshire NHS Trust, Macclesfield District General Hospital, Macclesfield, United Kingdom.
}

\begin{abstract}
Corresponding Author:
Dr MA Gok

Email: muhammed.gok@nhs.net

This is an Open Access article distributed under the terms of the Creative Commons Attribution License (creativecommons.org/ licenses/by/3.0).
\end{abstract}

Received : August 3, 2020

Accepted : December 19, 2020

Published : January 20, 2021

\begin{abstract}
Background: Emergency colorectal cancer accounts for $20 \%$ of colorectal cancer which usually presents late with obstruction, perforation, bleeding or abdominal pain. Small or large bowel obstruction occurs as a result of advanced proximal or distal colon cancer. Surgical treatment is mandatory to alleviate the intestinal obstruction, which may include resection of the underlying colon cancer. Case Report: This is a case report of splenic rupture resulting from an obstructed sigmoid cancer in a 62 year old patient. The patient presented with obstructive colorectal cancer symptoms. The patient suddenly developed severe abdominal pain and shock, CT scan diagnosis of splenic rupture resulted in early surgical intervention (splenectomy and Hartmann's resection). Conclusion: Rupture of the spleen is a rare complication of an obstructed colorectal cancer, which can present acutely in a shocked patient. Excessive traction on the spleno-colic ligament is believed to be the cause of subsequent splenic rupture.
\end{abstract}

Keywords: Abdominal Pain, Colonic Diseases, Sigmoid Neoplasms, Splenic Rupture, Splenectomy.

\section{Introduction}

Colorectal cancer is the $3^{\text {rd }}$ most common cancer and $2^{\text {nd }}$ most common cause of cancer related deaths amongst both males and females $[1,2]$. Colorectal cancer presents with colorectal symptoms (change in bowel habits, rectal bleeding, abdominal pain, tenesmus, weight loss, anaemia, and fatigue, abdominal or rectal mass). Diagnosis is made via endoscopy (colonoscopy, flexible sigmoidoscopy) and CT (computerized tomography) scan screening. Presentation of "emergency colorectal cancers" has remained at $20 \%$ of all colorectal cancers for the past decade [3]. Emergency colorectal cancers usually present with obstruction, perforation, bleeding and abdominal pain. Most "emergency colorectal cancers" present with small or large bowel obstruction, as a result of advanced proximal or distal large intestinal cancer which require mandatory treatment of obstruction.
The prevalence of splenic rupture in an obstructed colorectal cancer is not widely reported in the literature. Splenic rupture can be divided into two major categories: traumatic and non-traumatic. The mechanism of the majority of traumatic rupture continues to be motor vehicle injury, followed by direct abdominal blows. Traumatic injury occurs in $50-75 \%$, which can present early or delayed in the stable patient [4]. Non-traumatic splenic ruptures are uncommon, $7 \%$ are due to idiopathic causes, and 93\% attributed to pathological causes (tumours, infective, inflammatory, medical therapy, mechanical causes) $[5,6]$. Non-traumatic rupture carries a mortality of $12 \%$.

The aim of the study is to illustrate a case where a patient developed an atraumatic splenic rupture with an obstructed sigmoid cancer, which is under-reported in published literature. 


\section{Case Report}

A healthy fit 62-year-old man with a BMI (body mass index) of 23.7 presented to accident and emergency department with 4 weeks history of lower abdominal pain, distended abdomen, constipation and one episode of vomiting. His symptoms worsened over 24 hours where abdominal pain was generalized. The patient opened his bowels a day prior to admission, which consisted of liquid faeces. He subsequently had absolute constipation. There was no history of recent abdominal trauma. Clinically he had distended abdomen and had diffuse tenderness. On admission he had tachycardia (110 bpm), a blood pressure of $(106 / 45 \mathrm{mmHg})$, low hemoglobin (124 g/L), high white cell count $\left(21.4 \times 10^{9} / \mathrm{L}\right)$, raised urea and creatinine (10.2 $\mathrm{mmol} / \mathrm{L}, 225$ $\mu \mathrm{mol} / \mathrm{L}$ respectively). Intravenous fluids were administered and a urinary catheter was inserted for fluid management and monitoring. Plain abdominal and chest x-ray were unremarkable.

An hour later there was deterioration in the patient condition; with a pulse rate of 134 bpm; blood pressure dropped to $97 / 71 \mathrm{mmHg}$ and low temperature $\left(35.2^{\circ} \mathrm{C}\right)$. An initial diagnosis of partial large bowel obstruction was made and an NG (nasogastric) tube was inserted. Despite fluid resuscitation (4 litres of intravenous fluids), he remained hypotensive $(87 / 64 \mathrm{mmHg})$ and his hemoglobin dropped to $71 \mathrm{~g} / \mathrm{L}$. Arterial blood gas demonstrated compensated metabolic acidosis $\mathrm{pH} 7.35 / \mathrm{HCO}_{3} 17.1 \mathrm{mmol} / \mathrm{L}$ with lactate of 7.5 $\mathrm{mmol} / \mathrm{L})$. An urgent non-contrast CT (eGFR 23) showed widespread large volume acute hemoperitoneum with evidence of splenic rupture [Fig.1,2]. Patient was prepared for an urgent exploratory laparotomy. On laparotomy 4 litres of frank blood with clots was found in the peritoneal cavity, a small tear at the lower pole of the spleen and obstructing mid-sigmoid colonic mass with distended large bowel. An emergency Hartmann's procedure with splenectomy and end colostomy was performed.

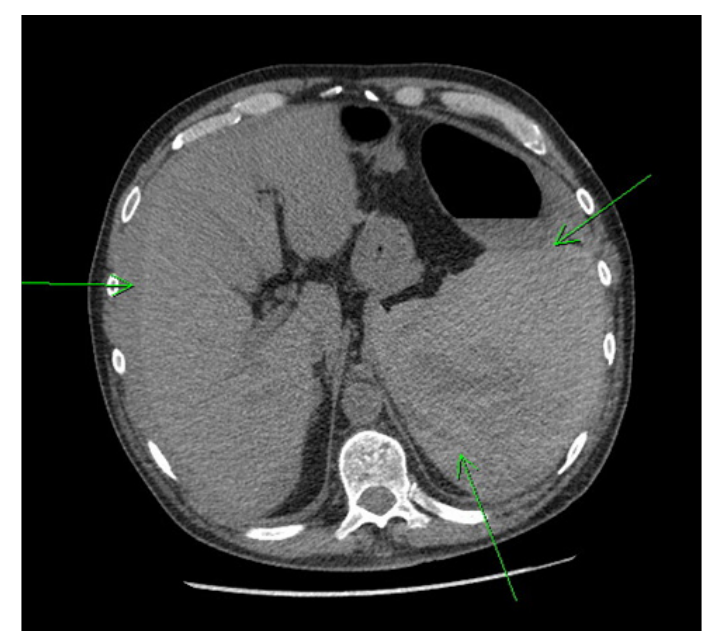

Fig.1: CT scan: Large volume acute haemo-peritoneum with evidence of splenic rupture.

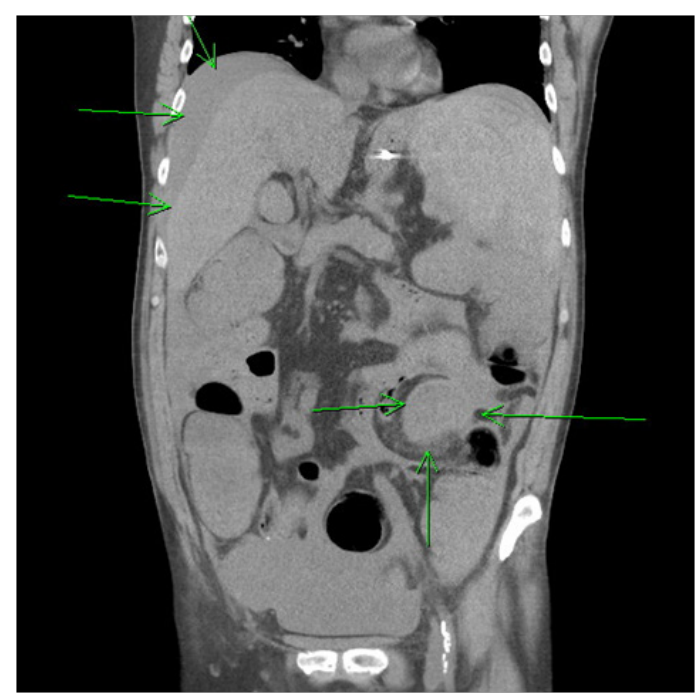

Fig.2: CT scan: Large volume acute haemo-peritoneum with evidence of splenic rupture and sigmoid tumor.

He was transferred to ITU (intensive treatment unit) for 2 days, and subsequent 7 days for ward level care. Prior to discharge a staging CT scan demonstrated a PE for which he was anticoagulated. He was subsequently discharged home with post-splenectomy vaccinations and prophylactic antibiotics. Histopathology of sigmoid demonstrated a moderately differentiated adenocarcinoma invading muscularis mucosa (Duke's B, pT3 pN0 p MX, 0/21 nodes) there was no venous or lymphatic invasion [Fig.3]. 
Histopathology of the spleen demonstrated increased thin walled vessels with focal capsular lower pole rupture, no evidence of malignancy or rupture to the splenic body [Fig.4]. Multidisciplinary review of his case was for surgical colorectal cancer surveillance programme. He subsequently had an uncomplicated reversal of colostomy 11 months post-operatively.

\section{Discussion}

Despite national initiatives to detect and diagnose colorectal cancer earlier, emergency colorectal cancers still accounts for $20 \%$ of all colorectal cancers [7-9]. Splenic rupture is a rare complication in an obstructed colorectal cancer, which is underreported [10]. Traumatic splenic rupture accounts for approximately $75 \%$ whilst atraumatic represents less than $12 \%$ of reported cases. Splenic rupture can occur in the presence of splenic neoplasms as either primary or secondary disease.

The presumed mechanism is believed to be caused by excessive traction on the splenocolic ligament causing avulsion and tear of splenic capsule, hematoma and subsequent spleen rupture as reported splenic rupture following colonoscopy [11]. This can be considered as internal trauma to a viscus organ. Awareness of such a mechanism of splenic rupture in an obstructed sigmoid cancer can lead to an earlier diagnosis in the absence of trauma. Splenic rupture in a stable patient can be treated with radiological interventional techniques (i.e. embolization of splenic artery) [12].

\section{Conclusion}

Atypical presentations could have adversely delayed the diagnosis of splenic rupture in an obstructed colorectal cancer. Serial clinical assessments and observations [MEWS (Modified early warning score), EWS (early warning score), etc.], supported by serological and radiological tests is crucial in the management of splenic rupture. Prompt resuscitation and radiological imaging facilitated an early diagnoses and surgical management.

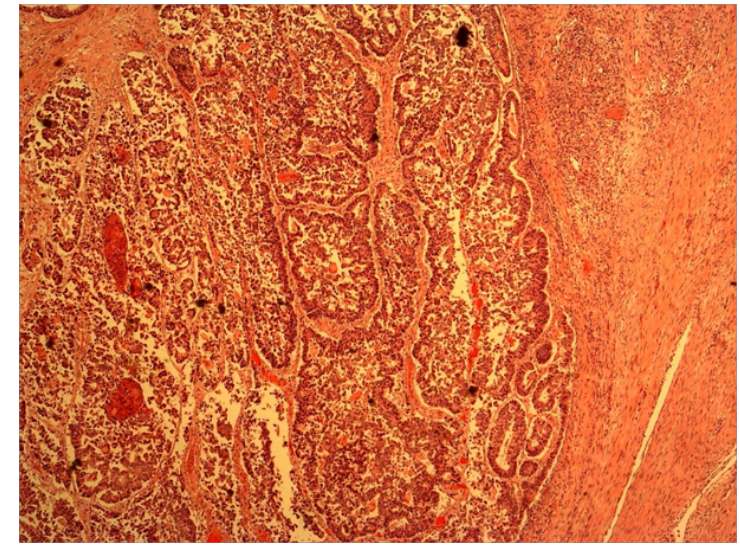

Fig.3: Histopathology of the splenic flexure showing adenocarcinoma of the colon.

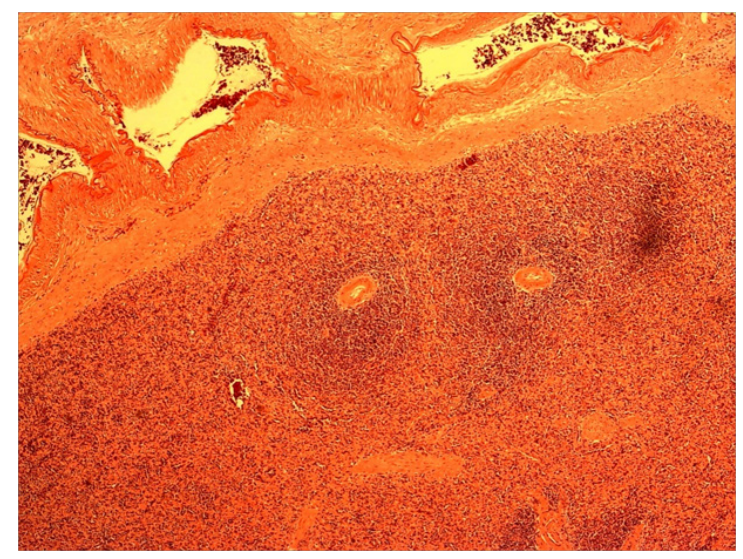

Fig.4: Histopathology of the excised spleen.

Contributors: OS: manuscript writing, patient management; FF, MAG: manuscript revision and patient management; MMS, UAK: critical inputs into the manuscript and patient management. MAG will act as study guarantor. All authors approved the final version of the manuscript and are responsible for all aspects of the study.

Funding: None; Competing interests: None stated.

\section{References}

1. Cancer Research UK. UK CR. Bowel cancer incidence statistics. Bowel cancer incidence statistics 2019. Available at: https://wwwcancerresearchukorg/healthprofessional/cancer-statistics/statistics-by-cancer-type/ bowel-cancer/incidence\#ref-. Accessed on December 19, 2020.

2. Cancer Research UK. The 20 Most Common Causes of Cancer Deaths, UK. Available at: https:// wwwcancerresearchukorg/health-professional/ 
cancer-statistics/mortality/common-cancerscompared\#heading-Zero. 2017. Accessed on December 19, 2020.

3. NBOCA. Annual Report 2019. National Bowel Cancer Audit Report. 2020; Available at: https://www.nboca.org. uk/content/uploads/2020/01/NBOCA-2019-V2.0.pdf. Accessed on December 19, 2020.

4. Raaijmakers C, Lohle P, Lodder P, de Vries J. Quality of life and clinical outcome after traumatic spleen injury (SPLENIQ Study): Protocol for an observational retrospective and prospective cohort study. JMIR Res Protoc. 2019;28(5):e12391.

5. Tunçyürek Ö, Tunçyürek P, Ertekin E, Gök M, Nevai E, Döger F, et al. Pathological rupture of the normal spleen: Review with the literature. Int J Surg Case Rep. 2016;26:163-165.

6. Renzulli P, Hostettler A, Schoepfer A, Gloor B, Candinas D. Systematic review of atraumatic splenic rupture. $\mathrm{Br} \mathrm{J}$ Surg. 2009;96(10):1114-1121.

7. Wanis K, Ott M, Van Koughnett J, Colquhoun P, Brackstone M. Long-term oncological outcomes following emergency resection of colon cancer. Int $\mathrm{J}$ Colorectal Dis. 2018;33(11):1525-1532.

8. Price J, Campbell C, Sells J, Weller D, Campbell H, Kenicer $\mathrm{M}$, et al. Impact of UK colorectal cancer screening pilot on hospital diagnostic services. J Public Health (Oxf). 2005;27(3):246-253.

9. NBOCA. Annual Report 2018. National Bowel Cancer Audit. 2018; Available at : https://www.nboca.org.uk/ content/uploads/2018/12/NBOCA-annual-report2018. pdf. Accessed on December 19, 2020.

10. Ellerker A. Ruptured spleen; report of five cases. Br Med J. 1955;2(4932):180-182.

11. Abunnaja S, Panait L, Palesty J, Macaron S. Laparoscopic splenectomy for traumatic splenic injury after screening colonoscopy. Case Rep Gastroenterol. 2012;6(3):624628.

12. Crooker K, Howard J, Alvarado A, McDonald T, Berry $\mathrm{S}$, Green $\mathrm{J}$, et al. Splenic embolization after trauma: An opportunity to improve best immunization practices. J Surg Res. 2018;232:293-297. 